STOMACH

\title{
Challenge model for Helicobacter pylori infection in human volunteers
}

\author{
D Y Graham, A R Opekun, M S Osato, H M T El-Zimaity, C K Lee, Y Yamaoka, W A Qureshi, \\ M Cadoz, T P Monath
}

See end of article for authors' affiliations

.....................

Correspondence to: Dr D Y Graham, Veterans Affairs Medical Center, Rm $3 A-320$ (1 11 1D), 2002 Holcombe Boulevard, Houston, TX 77030, USA; dgraham@bcm.tmc.edu

Revised version received 18 February 2004 Accepted for publication 19 February 2004

\begin{abstract}
Background: A reliable challenge model is needed to evaluate Helicobacter pylori vaccine candidates. Methods: A cag pathogenicity island negative, OipA positive, multiple antibiotic susceptible strain of $H$ pylori obtained from an individual with mild gastritis (Baylor strain 100) was used to challenge volunteers. Volunteers received $40 \mathrm{mg}$ of famotidine at bedtime and $10^{4}-10^{10} \mathrm{cfu}$ of $H$ pylori in beef broth the next morning. Infection was confirmed by ${ }^{13} \mathrm{C}$ urea breath test $\left({ }^{13} \mathrm{C}-\mathrm{UBT}\right)$, culture, and histology. Eradication therapy was given four or 12 weeks post challenge and eradication was confirmed by at least two separate UBTs, as well as culture and histology.

Results: Twenty subjects (nine women and 11 men; aged 23-33 years) received a $H$ pylori challenge. Eighteen (90\%) became infected. Mild to moderate dyspeptic symptoms occurred, peaked between days 9 and 12 , and resolved. Vomitus from one subject contained $>10^{3}$ viable/ml $H$ pylori. By two weeks post challenge gastric histology showed typical chronic $H$ pylori gastritis with intense acute and chronic inflammation. The density of $\mathrm{H}$ pylori (as assessed by cfu/biopsy) was similarly independent of the challenge dose. A minimal infectious dose was not found. Gastric mucosal interleukin 8 levels increased more than 20 -fold by two weeks after the challenge.

Conclusion: Challenge reliably resulted in $\mathrm{H}$ pylori infection. Infection was associated with typical $\mathrm{H}$ pylori gastritis with intense polymorphonuclear cell infiltration and interleukin 8 induction in gastric mucosa, despite absence of the cag pathogenicity island. Experimental $H$ pylori infection is one of the viable approaches to evaluate vaccine candidates.
\end{abstract}

$\mathrm{H}$ elicobacter pylori is a major pathogen aetiologically associated with gastritis, peptic ulcer disease, gastric cancer, and primary gastric lymphoma. Worldwide it is one of the most common chronic infections and is responsible for tremendous morbidity and mortality. While significant progress has been made in the treatment of $H$ pylori infection with antibiotics, current treatments are complex and their effectiveness is being undermined by the increasing prevalence of antibiotic resistance. ${ }^{1}$ Despite the variable success of $H$ pylori treatment, no preventative strategies have yet proven effective. The high worldwide incidence of the infection points to the clear need for a prophylactic vaccine with the ultimate immunisation target population being children as $H$ pylori is typically acquired in childhood. Vaccine studies in animal models have proven that the concept of vaccination is possible and vaccine candidates against $H$ pylori are in development. ${ }^{2-13}$

$H$ pylori vaccine development requires clinical trials to determine the effectiveness of prophylactic immunisation. As no immunological surrogates for protective immunity have yet been identified, successful vaccine trials will require demonstration of protection against infection and/or the pathological consequences of infection in humans. We report the development of a reproducible model of artificial $H$ pylori infection in healthy adults infected with $H$ pylori. The model is designed to facilitate clinical development of candidate vaccines. The decision to begin the program with $H$ pylori naïve volunteers was based on the premise that prior $H$ pylori infection and immunological experience with $H$ pylori antigens may influence the outcome of artificial immunisation.

Prior to developing a human $H$ pylori infection model, we considered a range of ethical and scientific issues, including selection of a challenge strain with the lowest risk of inducing disease and the highest probability of cure after reaching the primary objective of inducing human infection. Risk factors for disease expression and spread of the challenge infection were also minimised. The protocol included stages: (a) identification of a challenge strain, (b) manufacture of the challenge strain under conditions acceptable for human use and performance of control tests, (c) regulatory approval for administration to human subjects, (d) identification of challenge candidates, (e) challenge with determination of infection status by clinical symptoms, ${ }^{13} \mathrm{C}$ urea breath test (UBT), biopsy for quantitative $H$ pylori culture and pathological score, and serology, (f) treatment with antibiotics, and (g) follow up to document bacteriological cure.

It was hypothesised that the infectious dose would be an important variable in future vaccine trials as too high a dose might overwhelm protective immunity. In the study reported here, we performed preliminary dose-response studies to estimate the minimum dose of $H$ pylori required to establish human infection.

\section{METHODS}

\section{$H$ pylori strain}

$H$ pylori strains containing the cag pathogenicity island are associated with increased interleukin (IL)-8 production and inflammation, and an increased risk of a symptomatic outcome such as peptic ulcer or gastric cancer. However, as strains lacking the cag pathogenicity island are not devoid of

Abbreviations: ${ }^{13} \mathrm{C}-\mathrm{UBT},{ }^{13} \mathrm{C}$ urea breath test; IL, interleukin; OD, optical density; BCS, Baylor challenge strain 
risk of developing these diseases, there is no evidence that there is a "safe" H pylori infection. To minimise the risk of a symptomatic outcome in the very unlikely event that successful cure of the infection could not be achieved, we choose to use a cagA negative test strain recovered from a healthy volunteer with mild superficial gastritis and negative tests for hepatitis, syphilis, and human immunodeficiency virus (table 1). In addition, the strain used in this study was shown to be susceptible in vitro to all of the antimicrobials used in current anti- $H$ pylori therapy and the infection was cured with one course of therapy. The strain was also characterised with relation to putative virulence factors, including the whole cag pathogenicity island, vacA genotypes, ice $A$ alleles, babA2 status, and oipA status using polymerase chain reaction genotypes and sequencing. ${ }^{14-18}$ CagA protein was also examined by immunoblotting, as previously described. ${ }^{19}$ Vacuolating cytotoxin was measured by Hela cell assay, as previously described. ${ }^{15}$

\section{The Baylor strain of $\boldsymbol{H}$ pylori}

On primary isolation from the donor, individual colonies from the antrum and corpus were picked, suspended in $100 \mu \mathrm{l}$ of Brucella broth (Difco Laboratories, Detroit, Michigan, USA) with $5 \%$ heat inactivated calf serum (derived from certified US herds, HyClone Laboratories, Logan, Utah, USA), and plated onto Mueller Hinton agar (Difco) plates containing $5 \%$ heat inactivated calf serum. All plates were incubated for five days in a restricted use $\mathrm{CO}_{2}$ incubator at $37^{\circ} \mathrm{C}$ under $12 \% \mathrm{CO}_{2}$. Bacterial growth was tested to confirm their identity as $H$ pylori by positive reactions to oxidase, catalase, and urease, and by cellular morphology and Gram stain. Once confirmed, growth (Pl) was harvested into individual tubes of $1 \mathrm{ml}$ Brucella broth containing 20\% glycerol (Fisher Scientific, Houston, Texas, USA) and frozen at $-70^{\circ} \mathrm{C}$.

Each individual isolate $(500 \mu \mathrm{l})$ was aspirated from each tube and mixed to produce the stock challenge strain. The stock challenge strain was transferred onto fresh agar plates to produce the master cell bank stock cultures (P2). The master cell bank was transferred once more to fresh medium to produce the working cell bank (P3) under GMP conditions and this was used to produce fresh inoculum for each challenge (P4). The strain was designated as the Baylor challenge strain 100 (BCS 100; ATCC BAA-945).

Table 1 Characteristics of the donor and test strain of Helicobacter pylori

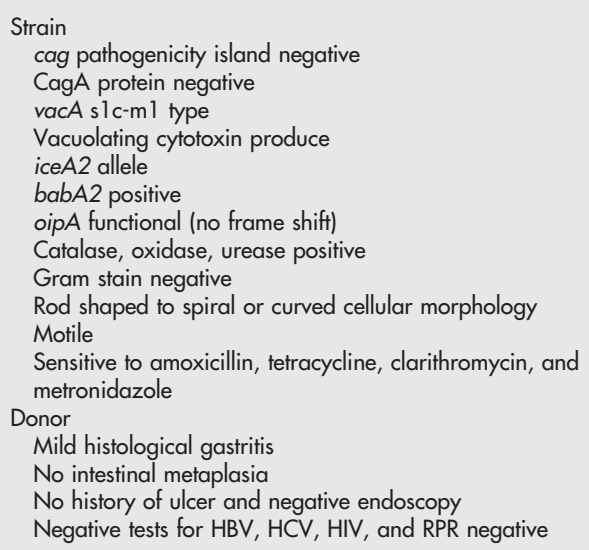

HBV, hepatitis B virus; HCV, hepatitis C virus; HIV, human immunodeficiency virus; $R P R$, rapid plasma repgin.

\section{Antibiotic susceptibility testing of the donor and challenge isolates}

The Etest (AB Biodisk, Solna, Sweden) was used to determine the antibiogram of the donor isolate and the manufactured challenge isolate used to infect the volunteers. The Etest was performed according to the manufacturer's instructions. Antibiotics tested included amoxicillin, clarithromycin, metronidazole, and tetracycline. All inoculated plates were incubated for three days at $37^{\circ} \mathrm{C}$ under $12 \% \mathrm{CO}_{2}$. The Etest minimum inhibitory concentration values were defined as the intercept of the elliptical zone of growth inhibition with the graded Etest strip, as per the manufacturer's instructions.

\section{Subject population experiment A (dose ranging)}

Subjects were aged $18-50$ years and in good health, as determined by medical history, physical examination, and clinical laboratory studies. Other criteria included no evidence of current or prior $H$ pylori infection by negative $H$ pylori serological test. (FlexSureHP; SmithKline Diagnostics Inc., San Jose, California, USA), negative ${ }^{13} \mathrm{C}$-UBT, normal upper gastrointestinal endoscopy with biopsies of the gastric antral and corpus mucosa being histologically normal and negative for $H$ pylori by Genta stain, culture, and rapid urease testing (hpfast; GI Supply, Camp Hill, Pennsylvania, USA). Female subjects were required to have a negative serum pregnancy test and use approved birth control throughout the study.

Exclusion criteria included living with, or having daily contact, with children aged 12 years or younger at home, school, day care, or equivalent facilities. Prohibition of contact with small children was based on epidemiological evidence that childhood is the time of greatest risk of developing $H$ pylori infection and thus children might have increased susceptibility to infection. ${ }^{20}$ Additional exclusion criteria included history of major gastrointestinal surgery, peptic ulcer disease, gastrointestinal haemorrhage, gall bladder disease, inflammatory bowel disease, frequent diarrhoea or irritable bowel syndrome, positive serology for human immunodeficiency virus, hepatitis B surface antigen, hepatitis C, or syphilis, regular use of corticosteroids, immunosuppressives, non-steroidal anti-inflammatory drugs, or anticoagulants, history of specific allergy to any of the drugs used to cure the infection, use of proton pump inhibitors, $\mathrm{H}_{2}$ receptor antagonists, bismuth, metronidazole, tetracycline, penicillin, or macrolide antibiotics within four weeks of study entry, a medical condition where there is anticipated use of antibiotics during the course of the study (approximately eight months), and finally, a history of bleeding diathesis that might increase the risk of gastric biopsy.

\section{Preparation of the inoculum}

The inoculum was prepared by seeding $100 \mu \mathrm{l}$ of frozen stock to 8-10 Mueller-Hinton agar plates containing $10 \%$ heat inactivated calf serum. Plates were incubated for five days at $37^{\circ} \mathrm{C}$ in a gas pak jar containing a CampyPak Plus (Becton Dickenson BBL, Cockeysville, Maryland, USA) gas generating envelope. Growth was harvested in Brucella broth and cell density was determined by measuring optical density (OD) at $550 \mathrm{~nm}$. Seed inoculum was added to $50 \mathrm{ml}$ of Brucella broth supplemented with $10 \%$ calf serum to achieve an $\mathrm{OD}_{550}$ of 0.1 (approximately $1 \times 10^{7}$ cells $/ \mathrm{ml}$ ). The inoculated flasks $\left(150 \mathrm{~cm}^{2}\right.$ tissue culture flasks; Corning Costar Corp., Cambridge, Massachusetts, USA) were loosely capped, then placed in a gas pak jar containing gas generating envelopes, and incubated overnight at $37^{\circ} \mathrm{C}$.

The overnight culture was tested for purity by Gram stain and for motility by hanging drop and $\mathrm{OD}_{550}$ was determined. A minimum $\mathrm{OD}_{550}$ of 0.2 must be achieved for the challenge 
to proceed. The contents of the flasks were distributed in sterile $50 \mathrm{ml}$ centrifuge tubes (Fisher) and centrifuged for 20 minutes at $3000 \mathrm{rpm}$. The bacterial pellet was gently resuspended in sterile saline and recentrifuged. The washed bacterial pellet was resuspended in College Inn Beef Broth (Nabisco Inc., East Hanover, New Jersey, USA) to a final concentration equivalent to an $\mathrm{OD}_{550}$ of 0.1 . Selection of this beef broth was made after a comparative analysis of stability of the challenge strain in a variety of comestible products. Cell density was adjusted to accommodate the dosing schedule (range $10^{4}-10^{10}$ cells total challenge dose). The beef broth containing the bacterial inoculum $(10 \mathrm{ml} / \mathrm{chal}-$ lenge) remained on ice until used for the challenge (within 20-30 minutes of preparation). Quantitative culture of the inoculum was performed to ascertain the actual concentration of bacteria administered per challenge.

\section{Clinical protocols}

The challenge study was divided into two phases: a dose ranging study (study A) and a dose validation study (study B). The first study phase was designed to show proof of concept and to refine the study to optimal conditions. The basic methodology for the two phases was similar with the exception that in study $\mathrm{B}$, the challenge dosages of $H$ pylori administered were lower and the infection was treated with antibiotics after one month. Each study consisted of a screening period, $H$ pylori challenge, post challenge follow up, antibiotic treatment, and follow up after therapy.

Subjects enrolled in study and who met the eligibility criteria received the BCS 100 cag pathogenicity island negative strain of $H$ pylori orally. On the evening prior to challenge, each subject received $40 \mathrm{mg}$ of famotidine to suppress gastric acidity. Subjects fasted for two hours after ingestion of the challenge dose.

In study A, subjects were assessed daily for two weeks after receiving the challenge strain and frequently thereafter.

${ }^{13} \mathrm{C}$-UBT and FlexSure IgG serology test were done on days 7,15 , and 90, with gastroscopy after two and 12 weeks. Infected subjects received eradication therapy on day 30 (five subjects) or on day 90 (four subjects). Eradication therapy consisted of quadruple therapy consisting of $20 \mathrm{mg}$ of omeprazole in the morning, $500 \mathrm{mg}$ of metronidazole three times daily, $500 \mathrm{mg}$ of tetracycline, and two tablets of PeptoBismol four times daily, all for 14 days. The importance of compliance with treatment was emphasised during visits and reinforced daily. Most subjects spoke to one of the investigators daily, exchanged emails, or both, on a daily basis. All subjects completed diaries and returned empty treatment medication bottles that were labelled for each patient. Compliance was determined by pill count.

Subjects enrolled in study B received one of three dosage levels $\left(\sim 10^{4}, \sim 10^{5}\right.$, and $\sim 10^{6}$ organisms $)$ of the challenge strain of $H$ pylori orally. Subjects were then assessed daily for four weeks. Eradication therapy consisted of triple therapy (30 mg lansoprazole, $500 \mathrm{mg}$ clarithromycin, and I g amoxicillin twice daily) for 14 days. ${ }^{13} \mathrm{C}$-UBTs were done on days 3 , 7, 14, and 28. Endoscopy with biopsy was done before therapy at four weeks and repeated to confirm cure at 12 weeks. ${ }^{13} \mathrm{C}$-UBT was repeated at approximately four weeks after treatment and the results confirmed approximately 12 weeks after treatment.

\section{Symptom assessment}

A symptom diary was completed by the subjects prior to challenge and continued for one month after challenge. A daily postcard containing symptom diary information was also provided and mailed or hand delivered by the subject daily to the investigators; non-receipt was followed up by a telephone call. Additionally, the study coordinator made a weekly phone call to or made personal contact with each subject to inquire about changes in symptoms. Subjects were encouraged to call/visit at any time regarding any change in clinical status.

\section{Histological assessment}

Endoscopy with biopsy was done before therapy and repeated to confirm cure on the schedule described above (see clinical protocols). At each gastroscopy, biopsies were taken for histological evaluation using large cup biopsy forceps (Radial Jaw; MicroVasive, Watertown, Massachusetts, USA, with an open diameter of $9.8 \mathrm{~mm}$ ) from predetermined locations from the greater and lesser curvatures of the antrum and corpus. Each specimen was placed in a separate bottle of formalin and routinely processed. Serial sections were cut at $5 \mu \mathrm{m}$ and stained with the Genta stain ${ }^{21}$ and El-Zimaity triple stain. ${ }^{22}$ Each specimen was reviewed by one pathologist and scored using a visual analogue scale from 0 (absent/normal) to 5 (maximal intensity) for acute inflammatory cells, chronic inflammatory cells, $H$ pylori, as well as for the presence or absence of lymphoid follicles. ${ }^{23}$

\section{$\mathrm{pH}$ of the gastric juice}

Gastric juice from several of the subjects was obtained during endoscopy and the $\mathrm{pH}$ of the specimens was determined using a pH meter (Radiometer America, Inc., Cleveland, Ohio, USA).

\section{Quantitative culture of biopsies}

Quantitative cultures were performed on all post treatment biopsies. Generally, two biopsies were taken from the antrum and two from the corpus. Specimens from each region were pooled in Brucella-glycerol broth $(1 \mathrm{ml})$ and homogenised by grinding between the frosted ends of two sterile glass slides. The homogenate was collected and placed back into the Brucella-glycerol broth. The tube was vortexed and $100 \mu \mathrm{l}$ of each homogenised tissue was used to prepare serial twofold dilutions in sterile saline. Diluted tissues $(100 \mu \mathrm{l})$ were plated onto Mueller Hinton calf serum agar plates and incubated at $37^{\circ} \mathrm{C}$ for seven days in a gas pak jar containing a CampyPak Plus (BBL) gas generating envelope. Following incubation, the emergent colonies on each plate were counted to ascertain the concentration of $H$ pylori per region of the stomach. Data are presented as number of colonies per biopsy.

\section{Cytokine levels in biopsy specimens}

Gastric mucosal specimens were immediately placed in $200 \mu \mathrm{l}$ of phosphate buffered saline ( $\mathrm{pH} 7.4)$, frozen on dry ice, and stored at $-80^{\circ} \mathrm{C}$ until use. Samples were homogenised using a tissue homogeniser (Kontes, Vineland, New Jersey, USA) and total protein in biopsy homogenate supernatants, obtained by centrifugation (10 $000 \mathrm{~g}$ for $10 \mathrm{~min}-$ utes), was measured by the Bradford assay (Bio-Rad, Richmond, California, USA); cytokine levels were measured by ELISA (R\&D Systems, Minneapolis, Minnesota, USA). In our laboratory, ELISA sensitivities of IL-1 $\beta$ and IL- 8 were approximately $5 \mathrm{pg} / \mathrm{ml}$ and those of IL- 6 were approximately $0.15 \mathrm{pg} / \mathrm{ml}$. Cytokine levels were expressed as $\mathrm{pg} / \mathrm{mg}$ protein.

\section{Statistical analyses}

Statistical analyses were done using SigmaStat v. 3.0 (SPSS, Chicago, Illinois, USA). Histological data are presented as the aggregate mean for the antrum and separately for the corpus. Results are presented as medians when data were not distributed normally and mean (SEM) when they were. Statistical analyses used paired parametric or non-parametric tests depending on whether the data were normally 
Table 2 Inoculum, duration of infection, and follow up for all patients given a challenge dose of Helicobacter pylori

\begin{tabular}{|c|c|c|c|c|}
\hline Age/sex & Subject No & Inoculum & $\begin{array}{l}\text { Cure of infection } \\
\text { (weeks) }\end{array}$ & Follow up* \\
\hline \multicolumn{5}{|c|}{ Study A: endoscopy at 14 days. } \\
\hline $29 \mathrm{M}$ & 101 & $1.7 \times 10^{10}$ & 12 & $4.5^{*}$ \\
\hline $25 \mathrm{~F}$ & 103 & $1.7 \times 10^{10}$ & 12 & 12 (UBT) \\
\hline $25 \mathrm{M}$ & 104 & $3.6 \times 10^{7}$ & 12 & 13 \\
\hline $29 M$ & 105 & $3.6 \times 10^{7}$ & 12 & 4 \\
\hline $24 \mathrm{~F}$ & 107 & $4.0 \times 10^{7}$ & 2 & 11 \\
\hline $23 \mathrm{~F}$ & 108 & $2.5 \times 10^{7}$ & 2 & 9.5 \\
\hline $34 \mathrm{M}$ & 109 & $1.1 \times 10^{6}$ & 2 & 10 \\
\hline $24 \mathrm{M}$ & 110 & $1.1 \times 10^{6}$ & 2 & 11 \\
\hline $33 \mathrm{~F}$ & 111 & $1.5 \times 10^{5}$ & 2 & 11 \\
\hline $24 M$ & 106 & $1.1 \times 10^{5}$ & Uninfected & 4 \\
\hline $23 \mathrm{~F}$ & 102 & $1.1 \times 10^{5}$ & Uninfected & 4 \\
\hline \multicolumn{5}{|c|}{ Study B: endoscopy at 30 days. } \\
\hline $29 \mathrm{M}$ & 205 & $1.0 \times 10^{4} / 5.0 \times 10^{4} \dagger$ & 4 & 3.5 \\
\hline $26 \mathrm{~F}$ & 206 & $1.0 \times 10^{4} / 1.4 \times 10^{5}$ & 4 & 4 \\
\hline $21 \mathrm{~F}$ & 207 & $1.0 \times 10^{4} / 1.2 \times 10^{5}$ & 4 & 4 \\
\hline $28 M$ & 201 & $2.3 \times 10^{5} / 2.3 \times 10^{5}$ & 4 & 3 \\
\hline $37 \mathrm{~F}$ & 203 & $2.3 \times 10^{5} / 2.3 \times 10^{5}$ & 4 & 3 \\
\hline $33 \mathrm{~F}$ & 202 & $2.3 \times 10^{5} / 2.3 \times 10^{5}$ & 4 & 3.5 \\
\hline $23 \mathrm{M}$ & 210 & $1.1 \times 10^{6} / 1.0 \times 10^{6}$ & 4 & 3.5 \\
\hline $26 M$ & 212 & $1.1 \times 10^{6} / 6.2 \times 10^{6}$ & 4 & 3.5 \\
\hline $22 \mathrm{~F}$ & 213 & $1.1 \times 10^{6} / 1.0 \times 10^{6}$ & 4 & 3.5 \\
\hline
\end{tabular}

*Last histological assessment (months post eradication therapy)

tlnoculum measured by back titration from actual inoculum.

distributed. Paired comparisons were made for those who had samples at both two and 12 weeks.

\section{RESULTS}

The Baylor challenge strain (BCS 100) was genotype cag pathogenicity island negative, and positive for vacA slc-ml, $i c e A 2$, and $b a b A 2$ with a functional oipA gene (for example, no frameshift in the $5^{\prime}$ region of the oipA gene) (table 1). The strain did not produce CagA protein but produced vacuolating cytotoxin.

\section{Subjects}

The study was performed at the VAMC and Baylor College of Medicine, Houston, Texas, USA. The study was conducted in accordance with the ethical principles that have their origins in the Declaration of Helsinki and with Good Clinical Practice rules. These practices included IRB procedures, informed consent, adverse event recording and reporting, inspection and audit preparation, and records retention. Representatives of the sponsor (OraVax, now Acambis) could inspect the

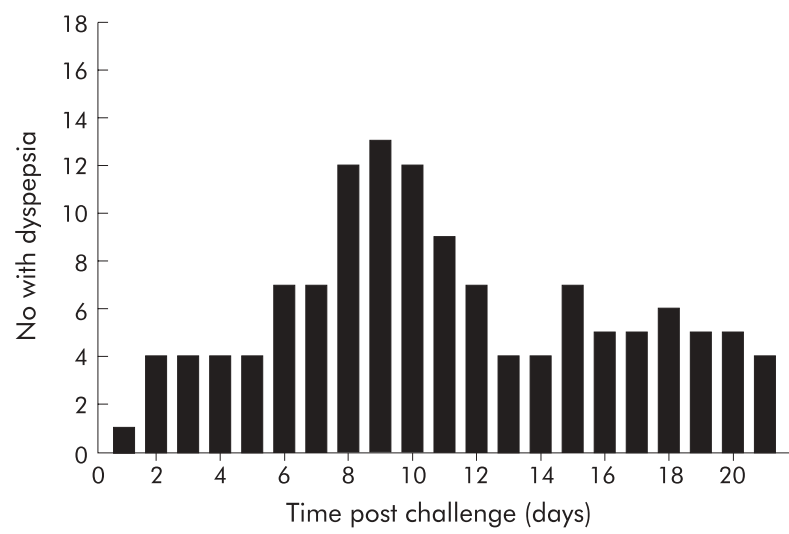

Figure 1 Frequency distribution of dyspepsia among 18 infected individuals during the first 21 days post challenge. The peak frequency of symptoms was between days 8 and 11 post challenge. documents and subject records at any time. All subject identities were kept confidential. Each subject was assigned a unique subject number which was used on the case report form instead of the subject's name. Each of the two protocols was approved separately by the institutional review boards at Baylor College of Medicine and the VA Medical Center. Protocol 2 (validation study) was conducted under an IND (BB-IND\#5853) reviewed and approved by the US Food and Drug Administration (FDA). The FDA reviewed the methods of manufacture and control of the $H$ pylori challenge strain and the clinical protocol. Results of the study were reported by OraVax (Acambis) to the FDA in the annual report filed to the IND. Volunteers reflected the composition of the medical centre and consisted of a group of knowledgeable individuals all of whom were college graduates. Volunteers were aware (before giving consent) of the remote but existing possibility of failure of eradication therapy and what this may mean in terms of potential long term consequences, namely the small possibility of developing peptic ulcer or gastric cancer. This group included eight junior or senior medical students, one $\mathrm{MD}-\mathrm{PhD}$ post doctoral fellow, three $\mathrm{PhD}$ graduate students in biomedical sciences, a PhD environmental scientist, and three technicians, including one with an MPH in medical or scientific fields and a graduate student in a medical field. There were three Asians, one Black, and one White Hispanic. Two were not US citizens, one had a permanent resident visa, and one had a visa for training. One was a post doctoral fellow (PhD) and the other a medical research technician with an MPH. Prior to enrolment, each subject received a copy of the consent documents to keep and discuss with whomever they chose. Subjects were reimbursed for participation at the same rates established for other volunteer studies involving endoscopy.

\section{Study A (proof of concept)}

Eleven subjects (five women and six men; aged 23-33 years) were challenged with the BCS $100 \mathrm{cag}$ pathogenicity island negative strain of $H$ pylori. Total doses of the inocula ranged from $1.7 \times 10^{10}$ cfu to $1.1 \times 10^{5} \mathrm{cfu}$ (table 2$)$. Nine $(82 \%)$ of 11 subjects became infected, as documented by positive ${ }^{13} \mathrm{C}$-UBTs at the earliest time point tested, seven days after 


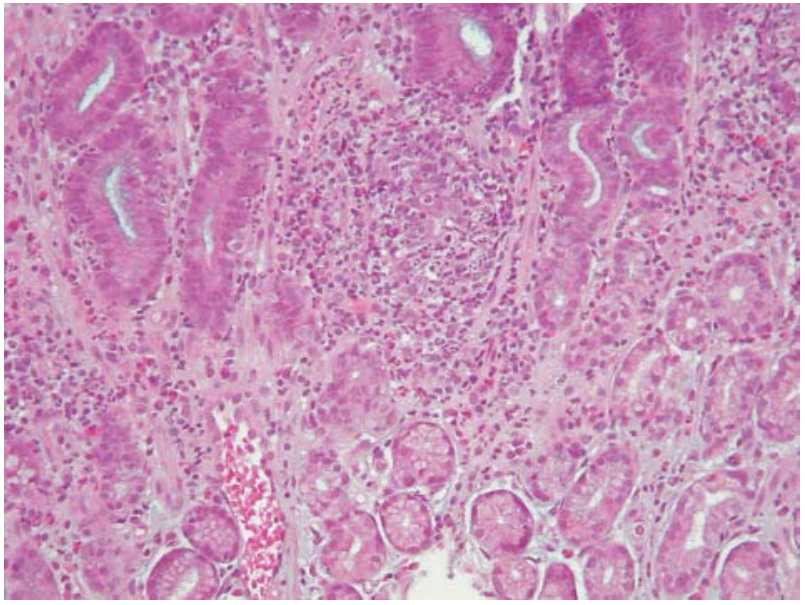

Figure 2 Two weeks post therapy. Typical Helicobacter pylori associated acute and chronic inflammation was observed two weeks after acquiring infection.

challenge. Two subjects who received the lowest inoculum were not infected.

The first four infected subjects were followed for 12 weeks before eradication therapy was given (group 1). Subsequently, subjects who were shown to be infected began therapy at the four week visit. All nine infected subjects were cured of infection following quadruple therapy. $H$ pylori eradication was determined by two separate negative ${ }^{13} \mathrm{C}$-UBTs and confirmed using biopsy for culture and histology in eight of the nine subjects.

\section{Study B: validation study}

In study $B$, nine subjects (four women and five men; aged $23-33$ years $)$ received one of three inocula $\left(1.0 \times 10^{6}, 2.3 \times 10^{5}\right.$, or $\left.3.25 \times 10^{4} \mathrm{cfu}\right)$. Back titration of the actual inocula showed that the expected inocula were delivered (table 2 ).

All subjects were breath test negative at three days post challenge. Seven of nine subjects developed positive ${ }^{13} \mathrm{C}$-UBTs at seven days post challenge, and an additional subject became positive at day 14 . One subject remained breath test negative throughout the study but proved to have been infected by histology and culture as endoscopy with histology and culture done at four weeks confirmed infection in all nine subjects. All subjects were cured of infection following triple therapy based on the results of two separate negative ${ }^{13} \mathrm{C}$-UBTs, as well as biopsy for culture and histology done three months after completing therapy or when first available.

\section{Symptoms \\ Study A}

Two subjects who were not infected did not have symptoms. Infected subjects developed variable gastrointestinal and constitutional symptoms. Dyspeptic symptoms were mild to moderate in severity and typically appeared during the first week after challenge and peaked between days 9 and 12 . Other recorded symptoms were: headache $(n=1)$, anorexia $(n=1)$, dyspepsia $(n=7)$, vomiting $(n=1)$, abdominal pain $(n=6)$, belching $(n=5)$, and halitosis $(n=3)$. In no instance did symptoms interfere with regular activities of daily living.

\section{Study B}

All subjects experienced dyspeptic symptoms that were mild to moderate in severity. Three subjects used antacid rescue medications. As with study A, symptoms typically first

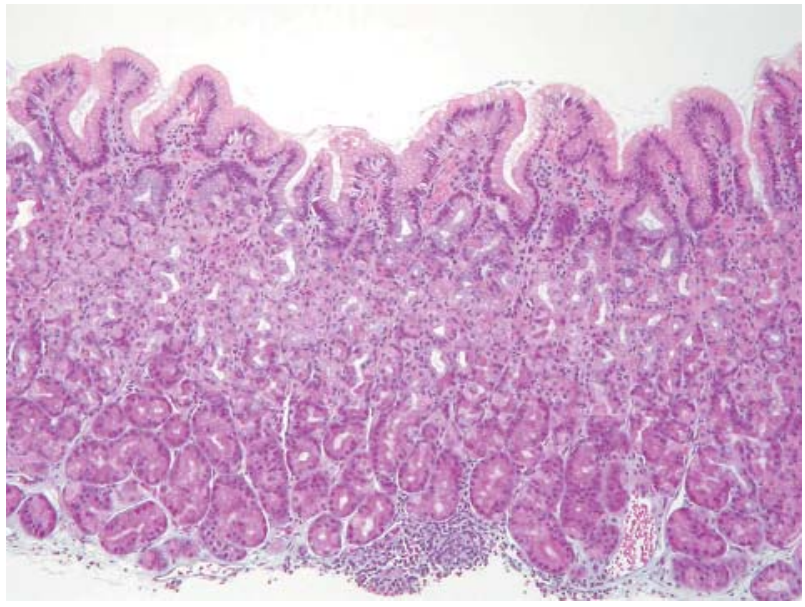

Figure 3 At baseline, organised lymphoid follicles (secondary lymphoid follicles) were not seen; only scattered isolated lymphoid infiltrates (primary lymphoid follicles) just above the muscularis mucosae were observed.

occurred sporadically after day 3, peaked in the second week, and remained relatively consistent for one week, and then resolved. Early symptoms included nausea and abdominal cramps, followed by feelings of abdominal fullness, belching, and mild decreased appetite after two weeks. There were two episodes of vomiting. One was concurrent with the onset of upper respiratory symptoms and believed to be related to a viral syndrome circulating in the community at the time of challenge. The other case was transient and more clearly associated with the study. Symptoms recorded included: chilly sensations $(n=1)$, headache $(n=3)$, anorexia $(n=5)$, dyspepsia $(n=6)$, vomiting $(n=2)$, cramping abdominal pain $(n=8)$, belching $(n=6)$, and halitosis $(n=1)$. In no instance did symptoms interfere with regular activities of daily living. Figure 1 shows the number of subjects with the most common symptom, dyspepsia, over the first three weeks post challenge for both study A and B combined.

\section{Follow up (study A)}

Gastroscopy was performed at 15 days (two weeks) in 10 subjects (one subject with a negative UBT refused repeat endoscopy). Four of the first six subjects, including four infected, were followed for 12 weeks before therapy thus providing two time points during infection for testing (two and 12 weeks). Uninfected patients were not treated for $H$ pylori eradication. The subject who declined follow up endoscopy had multiple negative UBTs extending over a period of 15 months; all were negative. The remaining subjects received anti- $H$ pylori therapy after four weeks.

Biopsies taken at two weeks showed the typical $H$ pylori histological pattern of infiltration, with acute and chronic inflammatory cells in biopsies from all nine infected subjects (fig 2). Follow up post cure showed absence of acute inflammatory cells. Organised lymphoid follicles were not observed in gastric biopsies before therapy. However, four of nine subjects that were successfully infected had small unorganised lymphoid aggregates below the glandular mucosa but above the muscularis mucosae at baseline (fig 3). Two had one such aggregate in the antrum, one had one aggregate in the corpus, and one had an aggregate in both the antrum and corpus. Another subject had a lymphoid aggregate with no apparent organisation in the mucosa of the corpus (fig 3). Organised lymphoid follicles were first observed at three months and were seen in antral biopsies in four subjects. Organised lymphoid follicles were not seen in any of the subjects who had small lymphoid aggregates 


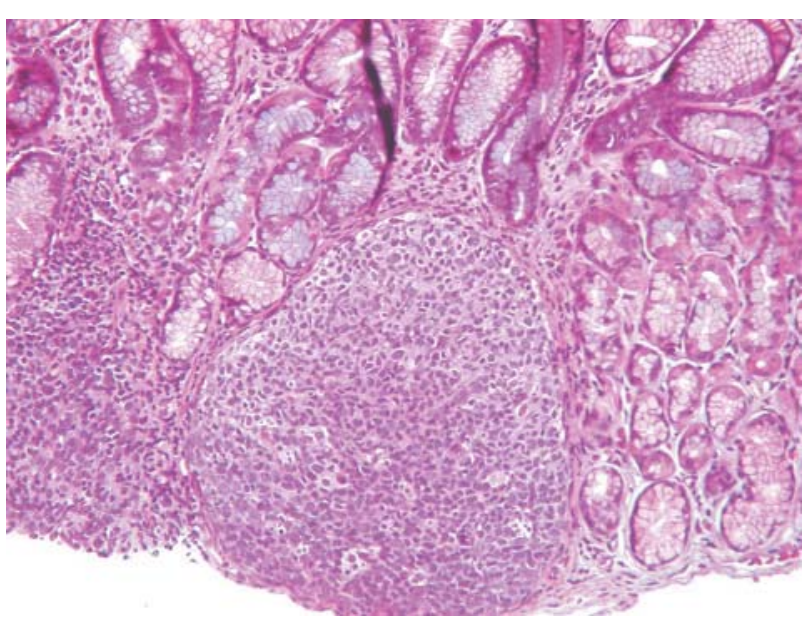

Figure 4 Post therapy. Remnants of organised lymphoid follicles were seen post therapy. In this image, the marginal zone is almost gone, and the lymphoid follicle centre is the only region observed.

below the glandular mucosa but above the muscularis mucosae at entry.

Infiltration of the lamina propria with chronic inflammatory cells decreased markedly after therapy. Remnants of previous follicles were noted to have a marked decrease in the marginal and mantle zones (fig 4). Nine were examined at one year post cure, the mononuclear cell infiltration decreased to a mean score of 2 in both the antrum and corpus, and remnants of lymphoid follicles were not seen. Small lymphoid infiltrates/and aggregates were present in six of the nine samples.

\section{Uninfected subjects}

Two subjects were not infected. In contrast with subjects who were infected, the first subject had four organised lymphoid follicles seen prior to challenge. No epithelial infiltration with polymorphonuclear leucocytes was observed pre or post challenge; the mononuclear score remained the same. The second subject had small lymphoid infiltrates just above the muscularis mucosae (one in the antrum and two in the corpus) prior to infection. Two weeks post challenge, epithelial infiltration with acute inflammatory cells (fig 5) was seen in one antral biopsy. $H$ pylori organisms were not

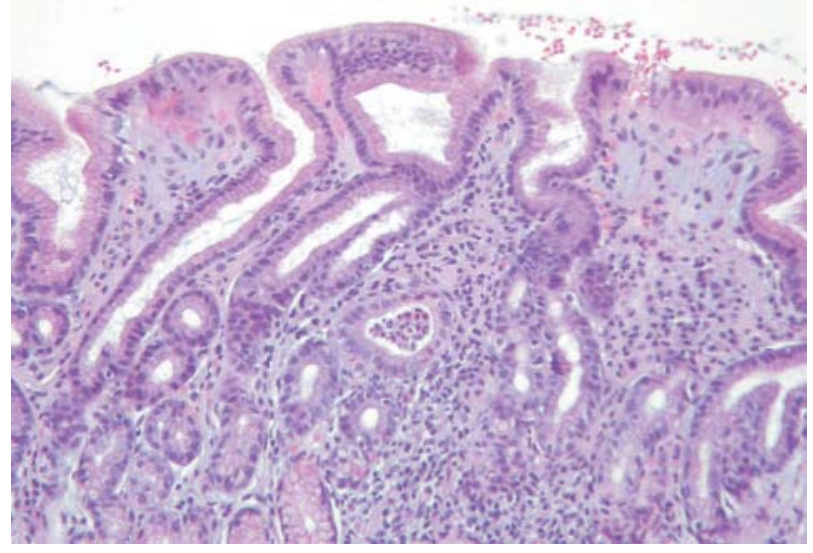

Figure 5 Two subjects remained uninfected post challenge. In one, two weeks post challenge, epithelial infiltration with acute inflammatory cells was seen in an antrum biopsy. Helicobacter pylori organisms were not identified up to 16 month post challenge.

\begin{tabular}{|lll|}
\hline Table 3 & Fasting gastric juice $\mathrm{pH}$ & \\
\hline Subject No & Sample period & $\mathrm{pH}$ value \\
\hline 101 & $+3 \mathrm{mo}$ & 2.11 \\
102 & $-2 \mathrm{wk}$ & 1.66 \\
& $+2 \mathrm{wk}$ & 1.57 \\
103 & $+3 \mathrm{mo}$ & 5.89 \\
104 & $+2 \mathrm{wk}$ & 7.41 \\
& $+3 \mathrm{mo}$ & 7.36 \\
105 & $+2 \mathrm{wk}$ & 3.26 \\
& $+3 \mathrm{mo}$ & 5.44 \\
106 & $-2 \mathrm{wk}$ & 2.16 \\
& $+2 \mathrm{wk}$ & 1.45 \\
107 & $+1 \mathrm{mo}$ & 4.0 \\
108 & $\mathrm{ND}$ & $\mathrm{ND}$ \\
109 & $\mathrm{ND}$ & $\mathrm{ND}$ \\
110 & $\mathrm{ND}$ & $\mathrm{ND}$ \\
111 & $+1 \mathrm{mo}$ & 2.69 \\
\hline
\end{tabular}

observed and the mononuclear cell infiltrate density was similar pre and post challenge. The subject was examined four and 16 months post challenge and he remained free of infection, and epithelial infiltration with polymorphonuclear leukocytes was not observed at follow up.

\section{Gastric juice pH}

Eight subjects had fasting gastric $\mathrm{pH}$ measured (table 3). Two of eight subjects had pre challenge gastric $\mathrm{pH}$ determinations performed, with an average pre challenge gastric juice $\mathrm{pH}$ of 1.9. Post challenge, gastric $\mathrm{pH}$ determination was performed at two weeks (four subjects), one month (two subjects), and at four months (four subjects). Between two weeks and one month post challenge, the average fasting gastric juice $\mathrm{pH}$ increased to 3.4 and 3.35, respectively. At three months post challenge, the average gastric $\mathrm{pH}$ increased further to 5.2.

\section{Follow up (study B)}

All nine individuals challenged became $H$ pylori infected, as shown by histology and culture. Overall the results of the histological analyses were similar (fig 6), with the exception that $H$ pylori density was statistically and significantly higher in the corpus compared with the antrum. Infiltration of polymorphonuclear cells into the gastric mucosa was intense in both the antrum and corpus.

\section{Quantitative culture of biopsies (studies A and B)}

In neither study was the density of $H$ pylori (as assessed by cfu/biopsy) in the antrum significantly different from that in the corpus, within or between studies (fig 7). Four individuals in study A were studied at both two and 12 weeks post challenge. $H$ pylori density in the antrum remained similar $\left(4.4(4.1) \times 10^{4} v 2.6(0.8) \times 10^{4}\right)(\mathrm{p}=0.69)$ and tended to increase in the corpus $\left(0.4(0.1) \times 10^{4} v 1.3(0.2) \times 10^{4}\right.$ $(\mathrm{p}=0.022)($ fig 8$)$.

\section{Culture of vomitus}

One patient and his spouse developed a brief gastroenteritis syndrome. He vomited once and the vomitus was collected. Samples $(100 \mu \mathrm{l})$ of the vomitus were plated directly onto Mueller Hinton agar plates containing 5\% calf serum and incubated in a gas pak jar that contained a CampyPak Plus gas generating envelope. Plates were incubated for seven days at $37^{\circ} \mathrm{C}$. Emergent colonies suggestive of $H$ pylori were picked and tested to confirm their identity (positive tests for oxidase, catalase, and urease, appropriate cellular morphology and Gram stain reaction). The spouse remained UBT negative and the episode was attributed to viral gastroenteritis that was prevalent in the community at that time. 

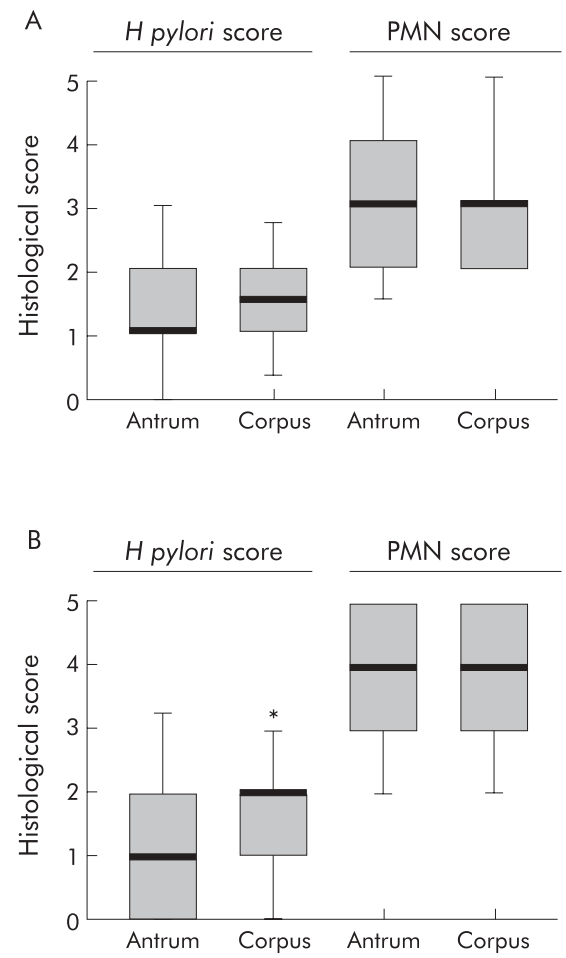

Figure 6 Box plots showing Helicobacter pylori density and polymorphonuclear (PMN) cell infiltrate scores for (A) study $A$, with biopsies at 14 days, and (B) study $B$, with biopsies at 30 days. The bold line represents the median value (50th percentile); shaded areas mark the 25th and 75th percentile points; and the two small horizontal lines show the 10th and 90 th percentiles.

\section{Cytokine levels in biopsy specimens}

Cytokine levels in antral biopsy specimens were evaluated in all subjects from study A. IL-1 $\beta$, IL-8, and IL-6 levels increased significantly two weeks after inoculation (IL-1 $\beta$ $9.2(2.5)$ to 49.1 (13.7) $\mathrm{pg} / \mathrm{mg}$ protein $(\mathrm{p}<0.05)$; IL-6 0.0 to $0.14(0.03) \mathrm{pg} / \mathrm{mg}$ protein $(\mathrm{p}<0.005)$; IL-8 4.1 (2.8) to 179.4 (40) $\mathrm{pg} / \mathrm{mg}$ protein $(\mathrm{p}<0.005)$. IL-8 levels increased more than 20 -fold in specimens obtained two weeks after inoculation. In corporal biopsy specimens, there was only a significant increase in IL-8 levels (4.6 (3.1) to 96.9 (31.2) $\mathrm{pg} / \mathrm{mg}$ protein) $(\mathrm{p}<0.05)$. After 12 weeks, IL-8 levels tended to decrease compared with two weeks, especially in antral specimens. In contrast, IL- $1 \beta$ levels were not different between two and 12 weeks. Despite the small number of samples available $(\mathrm{n}=4)$ there was a trend for IL-6 levels to increase (for example, more than fivefold in the antrum $(p=0.116)$ and 3.5 -fold in the corpus $(p=0.125))$ at 12 weeks compared with two weeks.

We examined cytokine levels for two subjects five months after antibiotic treatment. Post therapy cytokine levels had deceased to pre inoculation levels (IL-1 $\beta 17.9$ and $8.3 \mathrm{pg} / \mathrm{mg}$ protein, in the antrum and corpus, respectively; and IL- 6 and IL-8 levels were $0 \mathrm{pg} / \mathrm{mg}$ protein for both the antrum and corpus).

\section{DISCUSSION}

Clinical trials in which subjects are vaccinated and subsequently challenged to determine protective activity of vaccine candidates have been used extensively in the development of vaccines and drugs against both enteric and respiratory pathogens. These pathogens include trials with malaria, Q fever, Salmonella typhi, enterotoxigenic E coli, cholera, Shigella, Campylobacter jejuni, Norwalk virus, rhinoviruses, influenza

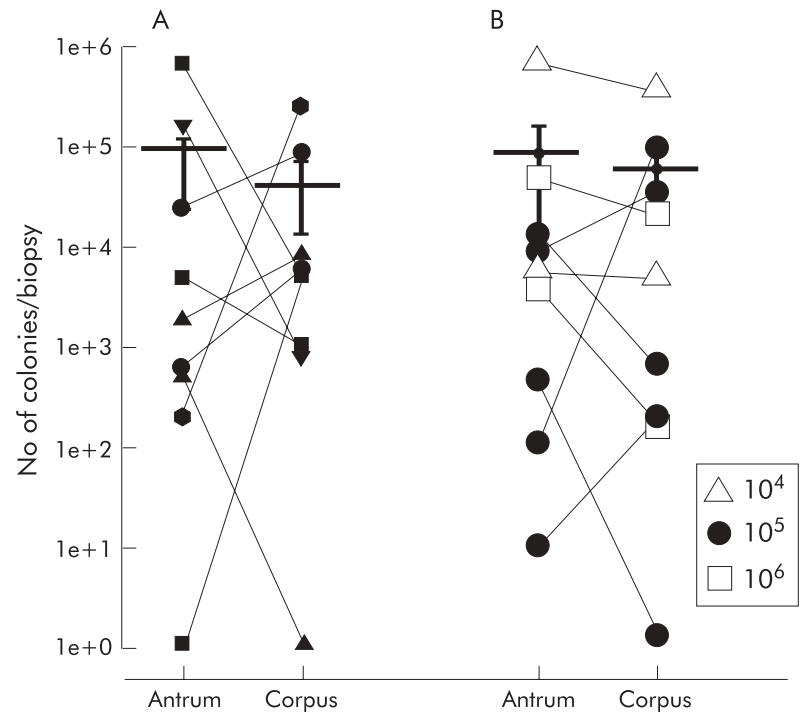

Figure 7 Helicobacter pylori density in the antrum and corpus, as measured using two antral and corpus biopsies. Data are expressed as cfu/biopsy. Mean (SEM) values are also shown for each group. There were no significant differences between the antrum and corpus or between study $A$ (with biopsy after 14 days) and study $B$ (with biopsy at 30 days). In (B) symbols represent an inoculum of $10^{4}, 10^{5}$, and $10^{6}$, as assessed by titration of the inoculum immediately post challenge.

virus, dengue viruses, sandfly fever virus, and respiratory syncytial virus. ${ }^{24-30}$ Our study shows that it is possible to reliably infect subjects with $H$ pylori and then cure the infection. The feasibility of performing successful $H$ pylori challenges with eradication was inferred from previous experiences where two individuals intentionally underwent self administered $H$ pylori challenge. Barry Marshall ingested $10^{9}$ cfu of $H$ pylori and developed a syndrome of dyspepsia that cleared after a few days. After confirming that gastric $H$ pylori infection had occurred, he took tinidazole, resulting in cure. $^{31}$ A second investigator in New Zealand ingested $3 \times 10^{5}$ cfu of $H$ pylori and also experienced a mild transient dyspeptic syndrome. After three years the infection was cured with antibiotics. ${ }^{32}{ }^{33}$ Other iatrogenically infected individuals have also been successfully treated, including one at this location. ${ }^{34}$

Safety is a primary concern in any challenge study in healthy subjects. In this study, as has been the practice with

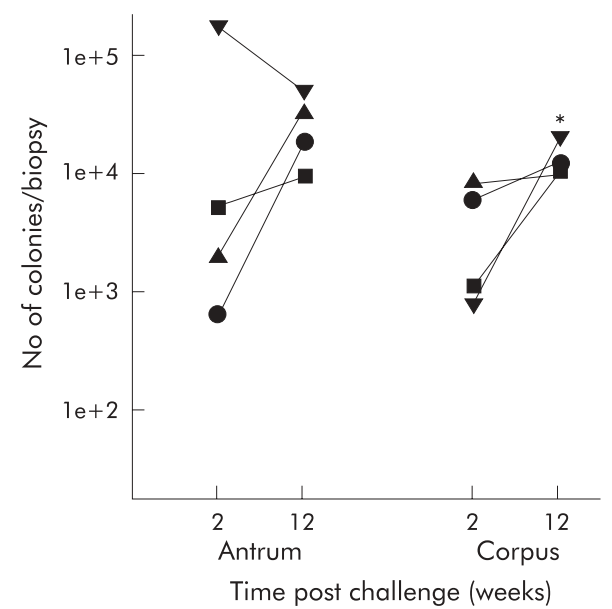

Figure 8 Helicobacter pylori density (cfu/biopsy) in the antrum and corpus two and 12 weeks post challenge. 
typhoid, shigellosis, and cholera, the experimental infection was cured with antibiotics to prevent chronic $H$ pylori infection. H pylori infection is typically acquired in childhood with the medically serious consequences such as peptic ulcer disease, mucosal atrophy, or neoplasia being preceded by many years (generally decades) of chronic inflammation induced by $H$ pylori. To minimise the risk of developing clinically significant disease, we used a short term model of infection during which subjects would be infected for only the time horizon of the proposed vaccine studies. The infecting strain chosen was selected to be one that, based on current concepts of the predictive value of putative virulence factors with regard to symptomatic outcomes, was of low virulence. In addition, the individual from whom the Baylor strain was obtained had only mild gastritis, the strain was susceptible to all of the antimicrobials used in current anti- $H$ pylori therapy, and the infection was eradicated from the donor. In this study, the success rate for antimicrobial therapy was $100 \%$ among those who became infected.

This study provided a unique opportunity to examine some of the early events in human $H$ pylori infection. Gastric mucosal biopsies taken two weeks after challenge revealed typical changes of both acute and chronic gastritis but without organised lymphoid follicles. This pattern of "acute chronic gastritis" occurred very rapidly such that by two weeks it may be impossible to distinguish recent infection from longstanding infection. The typical finding of an acute $H$ pylori infection is thought to be a neutrophilic gastritis. It seems likely that biopsies taken earlier would have revealed this finding. The appearance of typical organised lymphoid follicles was first seen in the antrum three months after challenge, suggesting that the finding of an organised lymphoid follicle indicates longstanding or chronic infection. Small unorganised lymphoid infiltrates that are present abutting the muscularis mucosae may also be seen in subjects with no histological, serological, or clinical evidence of prior $H$ pylori infection. It is not known whether these represent evidence of prior $H$ pylori infection.

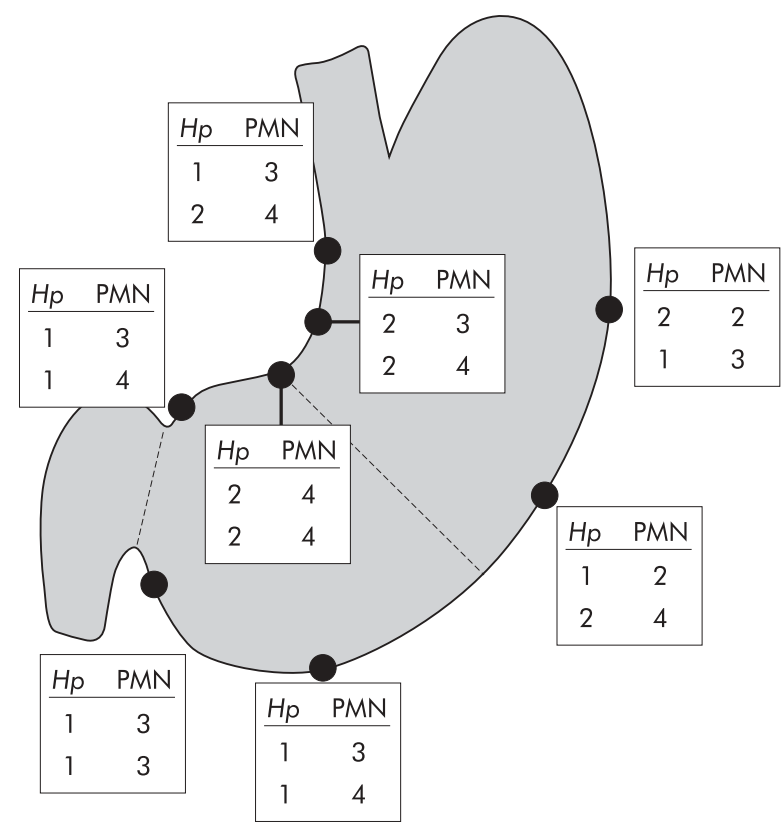

Figure 9 Results of histology at 14 (above) and 30 days (below) (study $A$ and study B, see text) are shown for each biopsy site. The highest density of Helicobacter pylori $(H p)$ and acute inflammatory cells was found at the gastric angle and approximately $2 \mathrm{~cm}$ proximal to it on the lesser curvature (sites A3 and B5). PMN, polymorphonuclear cells.
Despite the fact that the strain used lacked the cag pathogenicity island, there was intense infiltration of polymorphonuclear cells (figs 2,6) and an increase in mucosal IL-8 levels. IL-8 is a chemoattractant for polymorphonuclear cells and, in general, mucosal IL-8 levels correlate with polymorphonuclear cell infiltration. In this study, antral IL-8 levels increased rapidly within two weeks and decreased at 12 weeks although antral polymorphonuclear cell infiltration was similar at the two time periods. The marked and sustained polymorphonuclear cell infiltration seen in these experiments differs from what one might expect based on in vitro data. These studies have demonstrated that only $H$ pylori strains containing an intact cag pathogenicity island stimulate IL- 8 production when cocultured with gastric epithelial cells. ${ }^{35}{ }^{36}$ The strain used had a functional oipA gene, and the oipA gene product (the outer inflammatory protein) also has the ability to induce IL-8 from gastric epithelial cells in vitro. ${ }^{37} 38$ It is not known whether the marked acute inflammatory reaction was actually related to the presence of a functional oipA gene or whether a cagA negative strain with a non-functional oipA gene would have behaved similarly. Such experiments are unlikely to be done in humans.

Both individuals, cited above, who intentionally underwent self administered $H$ pylori challenge used drugs to reduce gastric acidity to theoretically improve the survival of $H$ pylori in the stomach. We also administered the $\mathrm{H}_{2}$ receptor antagonist, famotidine, the evening before challenge. It is not known whether this was required or what effect this may have had on the minimal infectious dose. The dose ranging study suggested that $10^{5} \mathrm{cfu}$ might be near the minimal infectious dose. In contrast, when nine individuals were challenged with inocula that ranged from $\sim 10^{4}$ to $\sim 10^{6} \mathrm{cfu}$, all became infected. The density of $H$ pylori in the gastric mucosa at either two or four weeks post challenge was not related to the size of the inoculum. It is likely that the threshold inoculum is less than $10^{4} \mathrm{cfu}$. The results are consistent with the need to sample both the antrum and corpus histologically. Sites with the highest density of $H$ pylori and acute inflammatory cells were both on the lesser curvature either at the gastric angle or just proximal to it (fig 9). This is consistent with previous studies evaluating $H$ pylori density and with identification of sites most likely to yield intestinal metaplasia, $H$ pylori, or a positive rapid urease test. ${ }^{39-42}$

Symptoms were common but in no case did they interfere with the tasks of daily living. Of note, $H$ pylori was easily cultured from the vomitus of one infected subject consistent with prior observations that $H$ pylori can be cultured from aspirates of gastric contents whether obtained by intubation or by vomiting. ${ }^{43}{ }^{44}$ Volunteers were cautioned about handwashing and other possible modes of transmission.

Preclinical studies in mice, ferrets, cats, and monkeys have generally shown that immunisation with experimental vaccines results in partial protection measured by a decrease in bacterial density in the stomach. ${ }^{9}{ }^{10-47}$ Bacterial density, assessed by quantitative culture post challenge, varied widely among individuals independently of the size of the inoculum (fig 7) suggesting that it would be unlikely to be possible to prove vaccine efficacy based solely on a consistent decrease in H pylori density.

In summary, this study shows that it is possible to reliably infect volunteers with $H$ pylori and then to eradiate the infection. The results of these studies suggest that 2-4 weeks post challenge is sufficient delay to confirm failure of protection. The fact that one subject did not develop a positive ${ }^{13} \mathrm{C}$-UBT throughout the period of infection shows that it will be important to obtain histopathological confirmation of failure of the challenge to produce infection. 
All individuals who received inocula of $10^{5}$ became infected, suggesting that $10^{5}$ would be a reasonable standard inoculum. No testing was done without prior administration of the $\mathrm{H}_{2}$ receptor antagonist famotidine and therefore is it not known whether this was actually necessary or whether a higher inoculum would be required.

\section{ACKNOWLEDGEMENTS}

This material was based on work supported in part by the Office of Research and Development Medical Research Service Department of Veterans Affairs and by Acambis, Inc, Cambridge, MA, USA, and by Aventis-Pasteur, Marcy l'Etoile, France.

\section{Authors' affiliations}

D Y Graham, A R Opekun, M S Osato, H M T El-Zimaity, C K Lee, Y Yamaoka, W A Qureshi, M Cadoz, T P Monath, VAMC and Baylor College of Medicine, Houston, TX, USA, Aventis-Pasteur, Marcy l'Etoile, France, and Acambis, Inc, Cambridge, Massachusetts, USA

\section{REFERENCES}

1 Miehlke S, Graham DY. Antimicrobial therapy of peptic ulcer. Int J Antimicrob Agents 1997;8:171-8.

2 Bonenfant C, Dimier-Poisson I, Velge-Roussel F, et al. Intranasal immunization with SAG1 and nontoxic mutant heat-labile enterotoxins protects mice against Toxoplasma gondii. Infect Immun 2001;69:1605-12.

3 Chen M, Lee A, Hazell S, et al. Immunization against gastric infection with Helicobacter species: first step in the prophylaxis of gastric cancer? Int J Med Microbiol Virol Parasitol Infect Dis 1993;280:155-65.

4 Corthesy-Theulaz IE, Hopkins S, Bachmann D, et al. Mice are protected from Helicobacter pylori infection by nasal immunization with attenuated Salmonella typhimurium phoPc expressing urease A and B subunits. Infect Immun 1998;66:581-6.

5 Czinn SJ, Nedrud JG. Oral immunization against Helicobacter pylori. Infect Immun 1991;59:2359-63.

6 Czinn SJ, Nedrud JG. Working towards a Helicobacter pylori vaccine. Gastroenterology 1999;116:990-3.

7 Del Giudice G. Towards the development of vaccines against Helicobacter pylori: status and issues. Curr Opin Invest Drugs 2001;2:40-4.

8 Ikewaki J, Nishizono A, Goto T, et al. Therapeutic oral vaccination induces mucosal immune response sufficient to eliminate long-term Helicobacter pylori infection. Microbiol Immunol 2000;44:29-39.

9 Kleanthous H, Myers GA, Georgakopoulos KM, et al. Rectal and intranasal immunizations with recombinant urease induce distinct local and serum immune responses in mice and protect against Helicobacter pylori infection. Infect Immun 1998;66:2879-86.

10 Lee CK, Soike K, Hill J, et al. Immunization with recombinant Helicobacter pylori urease decreases colonization levels following experimental infection of rhesus monkeys. Vaccine 1999;17:1493-505.

11 Satin B, Del Giudice G, Della B, V, et al. The neutrophil-activating protein (HPNAP) of Helicobacter pylori is a protective antigen and a major virulence factor. J Exp Med 2000;191:1467-76.

12 Bumann D, Holland P, Siejak F, et al. A comparison of murine and human immunoproteomes of Helicobacter pylori validates the preclinical murine infection model for antigen screening. Infect Immun 2002;70:6494-8.

13 Ruggiero P, Peppoloni S, Berti D, et al. New strategies for the prevention and treatment of Helicobacter pylori infection. Expert Opin Invest Drugs 2002;11:1127-38.

14 Mukhopadhyay AK, Kersulyte D, Jeong JY, et al. Distinctiveness of genotypes of Helicobacter pylori in Calcutta, India. J Bacteriol 2000;182:3219-27.

15 Yamaoka Y, Kodama T, Kita M, et al. Relationship of vacA genotypes of Helicobacter pylori to cagA status, cytotoxin production, and clinical outcome Helicobacter 1998;3:241-53.

16 Yamaoka Y, Kodama T, Gutierrez O, et al. Relationship between Helicobacter pylori iceA, cagA, and vacA status and clinical outcome: studies in four different countries. J Clin Microbiol 1999;37:2274-9.

17 Yamaoka Y, Orito E, Mizokami M, et al. Helicobacter pylori in North and South America before Columbus. FEBS Lett 2002;517:180-4

18 Gerhard M, Lehn N, Neumayer N, et al. Clinical relevance of the Helicobacter pylori gene for blood-group antigen-binding adhesin. Proc Natl Acad Sci U S A 1999;96:12778-83
19 Yamaoka Y, Kodama T, Kashima K, et al. Variants of the 3' region of the cagA gene in Helicobacter pylori isolates from patients with different $\mathrm{H}$. pyloriassociated diseases. J Clin Microbiol 1998;36:2258-63.

20 Malaty HM, Graham DY, Wattigney WA, et al. Natural history of Helicobacter pylori infection in childhood: 12-year follow-up cohort study in a biracial community. Clin Infect Dis 1999;28:279-82.

21 Genta RM, Robason GO, Graham DY. Simultaneous visualization of Helicobacter pylori and gastric morphology: a new stain. Hum Pathol 1994;25:221-6.

22 El-Zimaity HM, Ota H, Scott S, et al. A new triple stain for Helicobacter pylori suitable for the autostainer: carbol fuchsin/Alcian blue/hematoxylin-eosin. Arch Pathol Lab Med 1998;122:732-6.

23 El-Zimaity HM, Graham DY, Al-Assi MT, et al. Interobserver variation in the histopathological assessment of Helicobacter pylori gastritis. Hum Pathol 1996;27:35-41.

24 Black RE, Levine MM, Clements ML, et al. Experimental Campylobacter jejuni infection in humans. J Infect Dis 1988; 157:472-9.

25 Bartelloni PJ, Tesh RB. Clinical and serologic responses of volunteers infected with phlebotomus fever virus (Sicilian type). Am J Trop Med Hyg 1976;25:456-62.

26 Tacket CO, Binion SB, Bostwick E, et al. Efficacy of bovine milk immunoglobulin concentrate in preventing illness after Shigella flexneri challenge. Am J Trop Med Hyg 1992;47:276-83.

27 Mills J, Van Kirk JE, Wright PF, et al. Experimental respiratory syncytial virus infection of adults. Possible mechanisms of resistance to infection and illness. $\mathrm{J}$ Immunol 1971;107:123-30.

28 Hornick RB, Greisman SE, Woodward TE, et al. Typhoid fever: pathogenesis and immunologic control. 2. N Engl J Med 1970;283:739-46.

29 Sabin, AB. Research on dengue in World War II. Am J Trop Med Hyg 1952;1:30-40

30 Tigertt WD, Benenson AS. Studies on Q fever in man. Trans Assoc Amer Phys 1956:60:98-104

31 Marshall BJ, Armstrong JA, McGechie DB, et al. Attempt to fulfill Koch's postulates for pyloric Campylobacter. Med J Aust 1985;142:436-9.

32 Morris AJ, Ali MR, Nicholson GI, et al. Long-term follow-up of voluntary ingestion of Helicobacter pylori. Ann Intern Med 1991; 1 14:662-3.

33 Morris A, Nicholson G. Ingestion of Campylobacter pyloridis causes gastritis and raised fasting gastric $\mathrm{pH}$. Am J Gastroenterol 1987;82:192-9.

34 Graham DY, Alpert LC, Smith JL, et al. latrogenic Campylobacter pylori infection is a cause of epidemic achlorhydria. Am J Gastroenterol 1988;83:974-80

35 Censini S, Lange C, Xiang Z, et al. cag, a pathogenicity island of Helicobacter pylori, encodes type I-specific and disease-associated virulence factors. Proc Natl Acad Sci U S A 1996;93:14648-53.

36 Fischer W, Puls J, Buhrdorf R, et al. Systematic mutagenesis of the Helicobacter pylori cag pathogenicity island: essential genes for CagA translocation in host cells and induction of interleukin-8. Mol Microbiol 2001;42:1337-48.

37 Yamaoka Y, Kwon DH, Graham DY. A M(r) 34,000 proinflammatory outer membrane protein (OipA) of Helicobacter pylori. Proc Natl Acad Sci U S A 2000;97:7533-8

38 Yamaoka Y, Kita M, Kodama T, et al. Helicobacter pylori infection in mice: Role of outer membrane proteins in colonization and inflammation. Gastroenterology 2002; 123:1992-2004.

39 El-Zimaity HM, Al-Assi MT, Genta RM, et al. Confirmation of successful therapy of Helicobacter pylori infection: number and site of biopsies or a rapid urease test. Am J Gastroenterol 1995;90:1962-4.

40 Woo JS, El-Zimaity HM, Genta RM, et al. The best gastric site for obtaining a positive rapid urease test. Helicobacter 1996;1:256-9.

41 Genta RM, Graham DY. Comparison of biopsy sites for the histopathologic diagnosis of Helicobacter pylori: a topographic study of $\mathrm{H}$. pylori density and distribution. Gastrointest Endosc 1994:40:342-5.

42 El-Zimaity HM, Graham DY. Evaluation of gastric mucosal biopsy site and number for identification of Helicobacter pylori or intestinal metaplasia: role of the Sydney system. Hum Pathol 1999;30:72-7

43 Leung WK, Siu KL, Kwok CK, et al. Isolation of Helicobacter pylori from vomitus in children and its implication in gastro-oral transmission. Am J Gastroenterol 1999:94:2881-4

44 Parsonnet J, Shmuely H, Haggerty T. Fecal and oral shedding of Helicobacter pylori from healthy infected adults. JAMA 1999;282:2240-5.

45 Blanchard TG, Lycke N, Czinn SJ, et al. Recombinant cholera toxin B subunit is not an effective mucosal adjuvant for oral immunization of mice against Helicobacter felis. Immunology 1998;94:22-7.

46 Lee CK, Weltzin R, Thomas WD Jr, et al. Oral immunization with recombinant Helicobacter pylori urease induces secretory lgA antibodies and protects mice from challenge with Helicobacter felis. J Infect Dis 1995;172:161-72.

47 Weltzin R, Kleanthous H, Guirakhoo F, et al. Novel intranasal immunization techniques for antibody induction and protection of mice against gastric Helicobacter felis infection. Vaccine 1997;15:370-6. 